\title{
Cerebral haemorrhage and berry aneurysm: evidence from a family for a pattern of autosomal dominant inheritance
}

\author{
Roger Shinton, Jasbinder Palsingh, Bernard Williams
}

\begin{abstract}
Although families with several members suffering a cerebral haemorrhage have been reported previously, a family history of this stroke sub-type has not yet been firmly established as a risk factor for the disease. A family in whom cerebral haemorrhage has been clearly documented in five members, spanning three generations, is reported. In three a berry aneurysm was detected. There was no evidence of hypertension among any of the five cases. A sixth member of the family probably died of a cerebral haemorrhage but no necropsy was performed. By using established incidence rates for cerebral haemorrhage in the population, the probability of five such unrelated events arising in any family of similar size and longevity was calculated to be $4.9 \times 10^{-10}$. This family strengthens the case that an underlying genetic susceptibility does exist for a proportion of patients who have a cerebral haemorrhage. This susceptibility appears to be consequent upon berry aneurysm formation. The distribution of cases within this family is consistent with an autosomal dominant pattern of inheritance.
\end{abstract}

A familial tendency for stroke has been previously described but is not at present widely recognised. ${ }^{1}$ In the recent Royal College of Physicians report on stroke it was not listed among the risk factors for the disease. ${ }^{2}$ There have, however, been a number of reports of familial clusters of cerebral haemorrhage, the sub-type of stroke accounting for about $15 \%$ of cases. ${ }^{3-9}$ These families have included between two and four cases of proven cerebral haemorrhage, spanning one or two generations. Whether or not these clusters have been due to chance has not been formally assessed.

We report a family with cerebral haemorrhage documented at either necropsy, operation or by CT affecting five members over three generations (figure).

Case reports

Case I-2. A 53 year old female was found

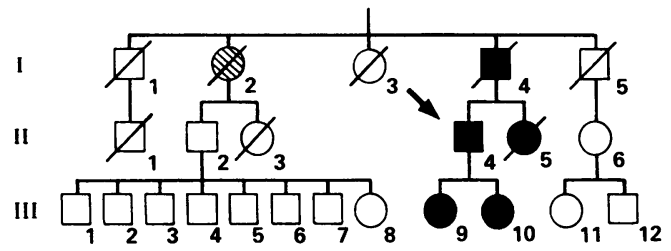

Figure The family pedigree $(\square$, males; $\bigcirc$,females; $\not$ $\varnothing$,deceased; $\square$, definite cerebral haemorrhage; suspected cerebral haemorrhage; $\rightarrow$ the propositus).

unconscious at her home in Smethwick in 1945. She died at home the same day. No necropsy was performed. The cause of death reported on the death certificate was: "cerebral haemorrhage, hypertension, arteriosclerosis". No further details were available.

Case I-4. A previously healthy 35 year old man was admitted to St Chad's Hospital, Birmingham in 1939 with sudden loss of consciousness. Lumbar puncture revealed heavily blood stained cerebrospinal fluid. The patient died on the day of admission. Necropsy revealed blood in the subarachnoid space but no berry aneurysm could be identified. The clinical and pathological information was obtained from a surviving autopsy report.

Case II-4. A 54 year old man, the propositus, was admitted to Dudley Road Hospital in 1989 with a history of sudden loss of consciousness associated with headache, photophobia and vomiting. On examination he had mild neck stiffness and bilateral extensor plantars but no other neurological abnormalities. His conscious level gradually returned to normal. CT revealed high density changes at the base of the brain suggesting subarachnoid haemorrhage. Carotid angiography revealed an aneurysm of the right middle cerebral artery which was subsequently clipped. The patient made a full recovery. Case II-5. A 26 year old woman was admitted to Dudley Road Hospital in 1958. She died 15 days later. No other history is available. Necropsy revealed a large intracerebral haemorrhage in the right frontal lobe with associated intraventricular haemorrhage. A ruptured aneurysm, $0.5 \mathrm{~cm}$ in diameter, was noted on the anterior cerebral artery, $1 \mathrm{~cm}$ from its origin. No subarachnoid haemorrhage was seen. The information was obtained from a surviving necropsy report.

Case III-9. A 29 year old woman was admitted to Dudley Road Hospital in 1988 follow- 
ing two generalised seizures witnessed by her husband. For three days before this she had noticed decreased power and sensation in her right arm. Examination revealed diminished power and sensation in her right arm but no other abnormalities. CT revealed a high density lesion in the left parietal region. Carotid and vertebral angiography was considered normal. A diagnosis of cerebral haemorrhage was made. She made a full recovery and has had no further seizures.

Case III-10. A 21 year old woman was admitted to Sandwell Hospital in 1986 with a history of sudden severe headache, photophobia, vomiting and transient confusion. No abnormal physical signs were noted but lumbar puncture revealed uniformly bloodstained cerebrospinal fluid. Angiography revealed a moderate sized aneurysm arising on the right internal carotid artery near the origin of the posterior communicating artery. The aneurysm was clipped and the patient made a full recovery.

Other members of the family illustrated in the figure, either alive or dead, have no history suggesting a cerebral haemorrhage. To date none has undergone cerebral angiography. Little is known of the cause of death of the parents of the first generation reported. There is no history of consanguinity in the family.

\section{Statistical assessment of the family clus- ter}

It is possible that this and other reported clusters of cerebral haemorrhage within families have arisen by chance. To assess this probability, the expected number of cases in a given cluster of people can be compared with the observed number of cases. The number of expected cases can be calculated by multiplying a previously established annual incidence rate by the person-years of the population (family) under consideration.

As the person-years of life (date of birth and possibly of death) and the diagnosis of cerebral haemorrhage have only been definitely established for the descendants of I-4, the analysis was restricted to this man and his descendants.

Data from the Oxford Community Stroke Project provide an annual incidence rate of well documented cerebral haemorrhage of 21 per $100000 .^{10}$ At the beginning of 1990 the family of I-4 (I-4, II-4, II-5, III-9 and III-10) had amassed a total of 169 person-years. The expected number of cases was therefore $169 \times 0.00021=0.036$. Five cases were observed. The Poisson distribution was used to assess the probability $\left(p_{n}\right)$ that in a given population $n$ events occurred when $u$ were expected $\left(p_{n}=u \times e^{-u} / n !\right)$. The values of $p$ for $\mathrm{n}=5,6,7, \ldots$ were summated to give a value of $4.9 \times 10^{-10}$ of observing five or more cases when 0.036 were expected.

The interpretation of probabilities quoted in association with clusters of cases, in either families or other populations is not straightforward. This family was clearly selected for reporting from many others who lacked the clustering of interest. In the United Kingdom roughly $1.5 \times 10^{7}$ "families" of similar size and longevity could have existed over the past 50 years (total population $\times 50$ years $/ 169$ person-years of the reported family). The probability that one or more of these will have yielded five or more cases is approximately $1.5 \times 10^{7} \times 4.9 \times 10^{-10}=0.007$. Thus despite the selectivity of the report, it remains highly improbable that the cluster arose by chance.

\section{Discussion}

We believe that the documentation of cerebral haemorrhage in this family and the remote likelihood of chance being responsible for the clustering, provides the best evidence to date that a genetic predisposition does exist for a proportion of patients suffering this type of stroke. The case is strengthened by the young age of most of the affected family members.

The family reported here provides support for the idea that the defect affecting the cerebral vasculature is inherited in an autosomal dominant pattern. This familial cluster of patients suffering a cerebral haemorrhage is the largest reported and the only cluster reported in which the diagnosis was well documented over three generations. A previously reported family of four cases of well documented cerebral haemorrhage, all due to berry aneurysm, involved only two generations, although the history suggested subarachnoid haemorrhage in the grandmother of one of those affected. ${ }^{4}$ Another previously reported family, involving four cases of proven berry aneurysm (three ruptured), also concluded that this vascular abnormality can be inherited in an autosomal dominant pattern.

The CT angiographic and necropsy findings on this family point to a common pathological defect predisposing to the haemorrhage. In three of the five members of the family a berry aneurysm was noted, one of which was only $0.5 \mathrm{~cm}$ in diameter. It would seem quite possible that in the other two patients a small aneurysm was destroyed at the time of the rupture.

There have been several reports of single families having between four and seven members with a well documented berry aneurysm. ${ }^{4-9}$ Although unruptured berry aneurysm may have a prevalence of as much as $5 \%$ of the population, these reports add weight to the concept that a proportion of aneurysms have a genetic predisposition, with the attendant risk of subsequent rupture.

The observation that three of the bleeds were subarachnoid and two intracerebral, does not fit well with the usual subclassification of cerebral haemorrhage into intracerebral and subarachnoid types. This family would support the idea that, in some instances, a similar pathogenesis may lie behind both subtypes. It may not be unreasonable therefore in some circumstances to group all cerebral haemorrhages together, particularly when assessing younger patients with an apparent familial pattern.

There was no evidence in this family that the predisposition to haemorrhage or berry 
aneurysm was associated with other congenital defects such as polycystic kidneys, Marfan's syndrome, tuberose sclerosis, Ehlers-Danlos syndrome or hereditary haemorrhagic telangectasia. ${ }^{11}$ The kidneys were reported as normal in the necropsy report on II-5 and in none of the cases was there any clear evidence of hypertension. A specific defect of the arterial wall may lie behind the tendency to arterial rupture in this family. The possibility of defective collagen synthesis requires further evaluation in this and other similar families. ${ }^{12}$ The possible contribution of potentially avoidable risks, such as smoking (patients I-4, II-4, II-5 and III-10) and use of the contraceptive pill (patients III-9 and III-10), in the pathogenesis of the reported cases of cerebral haemorrhage is unknown. It would, however, seem sensible to pay particular attention to these hazards in families who appear susceptible to this devastating illness.

We thank members of the family on which this report was based for their help. Dr Michael Hills gave helpful guidance on the statistical interpretation of the family cluster. Professor ER Hitchcock and Mr J Hamilton kindly gave permission to report the patients under their care.

1 Dyken ML, Wolf PA, Barnett HJM, et al. Risk factors in stroke. A statement for physicians by the subcommittee on risk factors and stroke of the stroke council. Stroke 1984;15:1105-11.

2 Royal College of Physicians Working Party. Stroketowards better management. London: Royal College of Physicians, 1989.

3 Bannerman RM, Ingall GB, Graf CJ. The familial occurrence of intracranial aneurysms. Neurology 1970;20:283-92.

4 Mellergard P, Ljunggren B, Brant L, Johnson U, Holtas S. HLA-typing in a family with six intracranial aneurysms. Brit J Neurosurg 1989;3:479-86.

5 Fox JL, Ko JP. Familial intracranial aneurysms. Six cases among 13 siblings. J Neurosurg 1980;52:501-3.

6 Fox JL. Familial intracranial aneurysms. Case report. $J$ Neurosurg 1982;57:416-7.

7 Evans TW, Venning MC, Strang FA, Donnai D. Dominant inheritance of intracranial berry aneurysm. BMJ 1981; 283:824-5.

8 Hashimoto I. Familial intracranial aneurysms and cerebral vascular anomalies. $J$ Neurosurg 1977;46:419-27.

9 Lozano AM, Leblanc R. Familial intracranial aneurysms. $J$ Neurosurg 1987;66:522-8.

10 Oxfordshire Community Stroke Project. Incidence of stroke in Oxfordshire: first year's experience of a community stroke register. $B M J$ 1983;287:713-7.

11 Weir B. Aneurysms affecting the nervous system. London: Williams and Wilkins, 1987

12 Neil-Dwyer G, Bartlett JR, Nicholls AC, Narcisi AC, Pope FM. Collagen deficiency and ruptured cerebral aneurysms. A clinical and biochemical study. $J$ Neurosurg 1983;59:16-20. 